\section{ORIGINAL RESEARCH}

J.Y. Chueh

A.K. Wakhloo

M.J. Gounis

\title{
Effectiveness of Mechanical Endovascular Thrombectomy in a Model System of Cerebrovascular Occlusion
}

BACKGROUND AND PURPOSE: A number of thrombectomy devices are currently undergoing clinical evaluation; meanwhile, various novel devices are under investigation. The aims of this study were to quantify flow restoration and the particle size distribution of the effluent pursuant to MET in an in vitro occlusion model.

MATERIALS AND METHODS: The model system was composed of 3 elements: an ICA/MCA replica, a clot model with mechanical properties similar to those of thrombi found in patients at risk of stroke, and a pulsatile flow loop. Different thrombectomy mechanisms including mechanical retrieval, aspiration, and waveguide induced cavitation were used. The efficacy end points were recanalization rate and amount of flow restoration. The risk of the embolic shower was assessed to evaluate device safety.

RESULTS: The recanalization rates were the following: Merci, 67\%; Solitaire, 100\%; Penumbra, 83\%; Enterprise, 17\%; and the waveguide, $0 \%$. In experiments in which recanalization was achieved, the amount of flow restoration for the Merci, Solitaire, and Enterprise devices was 100\%, 92\%, and $86 \%$, respectively. The mean sizes of generated small and large clot fragments were between 23 and 37 and 215 and $285 \mu \mathrm{m}$, respectively, depending on the device used. The Merci device generated the fewest number of large fragments compared with the Penumbra system $(P<.05)$ and Solitaire (not significant).

CONCLUSIONS: The risk of embolic shower was influenced by the mechanism of action for the thrombectomy device. Clinically reported recanalization rates for the Solitaire, Penumbra, and Merci devices were nearly identical in this model system, suggesting that this model may provide a predictive tool for preclinical evaluation of MET.

ABBREVIATIONS: $\mathrm{AIS}=$ acute ischemic stroke; $\mathrm{CCA}=$ common carotid artery; $\mathrm{ECA}=$ external carotid artery; $\mathrm{MERCI}=$ Mechanical Embolus Removal in Cerebral Ischemia; $\mathrm{MET}=$ mechanical endovascular thrombectomy

$\mathbf{P}$ rompt recanalization of the occluded vessel in AIS may reduce the amount of brain destined for infarction. ${ }^{1-3}$ Previous studies have shown that MET allows AIS treatment in patients contraindicated for treatment with intravenous recombinant tissue plasminogen activator. ${ }^{4,5}$ However, further improvement in the effectiveness and thus clinical outcome of MET devices is desired.

The feasibility of using the retrievable stent to extract the thrombotic material from an occluded vessel has been demonstrated in the porcine model. ${ }^{6}$ This technique is now being

Received November 30, 2011; accepted after revision February 9, 2012.

From the Department of Radiology, New England Center for Stroke Research, University of Massachusetts Medical School, Worcester, Massachusetts.

This work was supported by the National Institute of Biomedical Imaging and Bioengineering grant 5R21EB007767-03. We also acknowledge technology support from Philips Healthcare.

The content of this work is solely the responsibility of the authors and does not necessarily represent the official views of the supporting institutions.

Paper previously presented at: 49th Annual Meeting of the American Society of Neuroradiology, June 4-9, 2011, Seattle, Washington; 2011 Summer Bioengineering Conference, June 22-25, 2011, Farmington, Pennsylvania; and 2012 International Stroke Conference, February 1-3, 2012, New Orleans, Louisiana.

Please address correspondence to Matthew J. Gounis, PhD, Department of Radiology, Director, New England Center for Stroke Research, University of Massachusetts, 55 Lake Ave North, SA-107R, Worcester, MA 01655; e-mail: matt.gounis@umassmed.edu

Indicates open access to non-subscribers at www.ajnr.org

http://dx.doi.org/10.3174/ajnr.A3103

evaluated in several ongoing clinical trials. ${ }^{7,8}$ Sonographically driven waveguides have been used for thrombus-ablation applications in the peripheral vasculature. ${ }^{9,10}$ In the present work, this concept is expanded to the ablation of cerebrovascular occlusion, in which the vessel geometry is more complex and tortuous.

We hypothesized that an experimental model system of cerebrovascular occlusion can be built to allow quantification of the flow restoration and characterization of the distal emboli. With these preliminary data, the effectiveness and safety of different thrombectomy techniques can be assessed before their clinical use.

\section{Materials and Methods}

\section{A Model System of Cerebrovascular Occlusion}

An ICA/MCA replica with an ICA siphon average curvature of 0.4 $\mathrm{mm}^{-1}$, a diameter of $4.2 \mathrm{~mm}$, and length of $20.6 \mathrm{~mm}$ was constructed by using a small-batch processing method, as previously reported. ${ }^{11}$ The MCA diameter at the site of the occlusion measured $2.1 \mathrm{~mm}$. In brief, the geometry of the vasculature was obtained from brain MRA data and was used to create a mold with core-shell structure for silicone infusion. After dissolution of the mold, a layer of LSR topcoat (Momentive Performance Materials, Albany, New York) was applied to the inner wall of the ICA/MCA silicone replica to provide enhanced lubricity for the delivery of endovascular devices. To create the MCA occlusion, a thrombin-induced radiopaque bovine clot model (10 


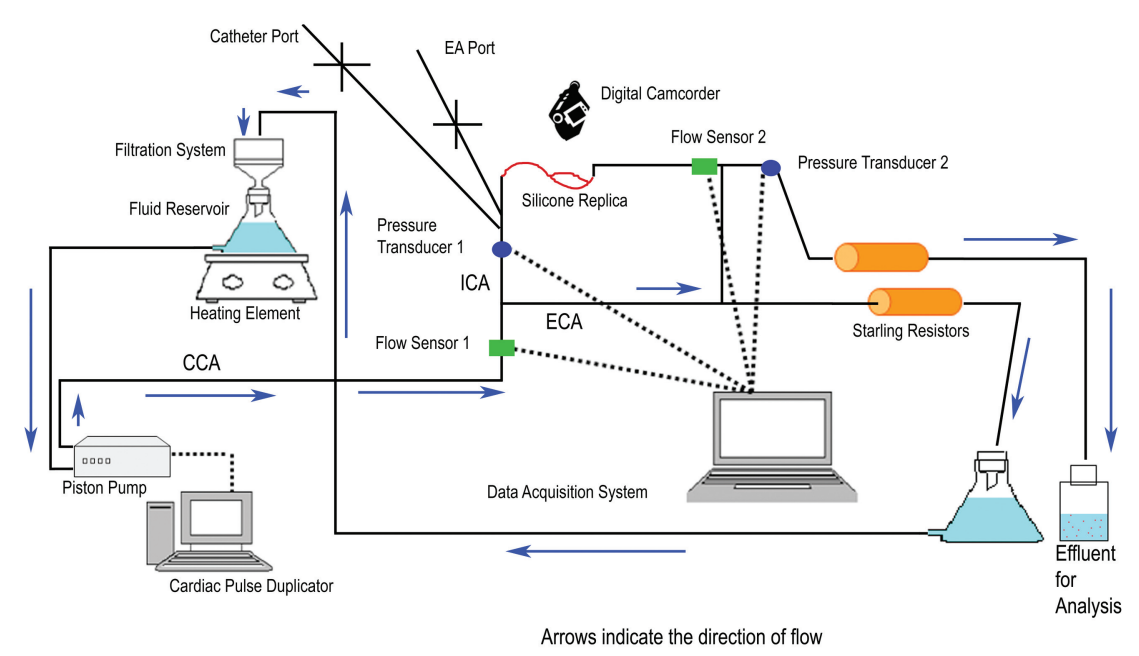

Fig 1. Schematic of the experimental system that includes ECA and ICA circulation loops. The flow through the ECA loop is filtered and recirculated, whereas the effluent of the ICA loop where the MET is performed is captured for particulate analysis. Location of the flow sensors and pressure transducers is noted. Use of an anastomosis between the dista ICA and ECA along with the Starling resistors maintains a physiologic pressure gradient across the MCA occlusion. $\mathrm{mm}$ in length) was delivered and carried with the flow, where it became lodged in the MCA. This particular clot model mimicked the thrombus extracted from the atherosclerotic plaques and was formed from a mixture of bovine blood, thrombin (2.5 NIH units $/ \mathrm{mL}$ blood $)$, and barium sulfate $(1 \mathrm{~g} / 10 \mathrm{~mL}$ blood $) .^{12}$

The flow loop (Fig 1) established in this study consisted of a programmable piston pump (Shelley Medical Imaging Technologies, Toronto, Ontario, Canada), a blood-mimicking fluid ( $60 \% / 40 \%$ by volume water/glycerin), custom-built Starling resistors chambers, and a data-acquisition system. Flow into the circulation loop as well as through the ICA/MCA replica was recorded digitally with sonographic flow probes (Transonic Systems, Ithaca, New York). Additionally, pressure was recorded proximal and distal to the site of occlusion by using variable reluctance differential pressure transducers (Validyne Engineering Corporation, Northbridge, California). A physiologically realistic flow waveform was generated by the pump, simulating human cerebrovascular hemodynamics. The blood analog fluid traveled through the external carotid artery and was directed to a filtration system before re-entering the reservoir; on the other hand, fluid traveling through the ICA loop was collected for particle analysis. The Starling resistors simulated the peripheral arterial resistance and controlled the pressure within the physiologic range. ${ }^{13}$ The data acquisition system (Dewetron, Charlestown, Rhode Island) recorded the real-time flow and pressure. It also incorporated the synchronized video captured by a digital camcorder.

\section{Thrombectomy Procedure}

The Merci L5 retriever (Concentric Medical, Mountain View, California), Enterprise stent (Codman Neurovascular, Raynham, Massachusetts), Solitaire FR revascularization device (ev3, Irvine, California), Penumbra 054 aspiration system (Penumbra, Alameda, California), and an ultrasound waveguide system (OmniSonics Medical Technologies, Wilmington, Massachusetts) were tested in this study. Each device was deployed according to the manufacturer's instructions.

To deliver the Merci retriever (helix loop of $4.5 \mathrm{~mm}$ in length and $2.5 \mathrm{~mm}$ in diameter), an $8 \mathrm{~F}$ balloon-guide catheter was positioned in the proximal ICA. Navigation of the Merci microcatheter 18L through the clot was performed over the wire under fluoroscopic navigation. The guidewire was exchanged for the Merci retriever, which was deployed beyond the occlusive clot. The corkscrew-shaped retriever engaged the clot and was pulled back into the guide catheter under proximal temporary balloon occlusion of the ICA. Aspiration through the balloon guide catheter was applied during clot retrieval.

The Enterprise stent ( $28 \mathrm{~mm}$ in length and $4.5 \mathrm{~mm}$ in diameter) has a closed-cell design and can be resheathed after up to $70 \%$ deployment. For stent-based thrombectomy, a $6 \mathrm{~F}$ guide catheter was placed in the proximal ICA. A $2.3 \mathrm{~F}$ microcatheter was navigated over the wire to the distal end of the occlusive clot. The guidewire was withdrawn followed by partial stent deployment within the occlusion for clot retrieval. During the retrieval, aspiration was applied through the central lumen of guide catheter. The procedure was performed without proximal flow arrest.

The Solitaire FR revascularization device $(20 \mathrm{~mm}$ in length and 4 $\mathrm{mm}$ in diameter) can be fully deployed, resheathed, and retrieved. The procedure used for the Enterprise deployment was applied to the Solitaire FR revascularization device, except that an $8 \mathrm{~F}$ balloon guide catheter was positioned in the ICA and inflated to temporarily arrest the proximal flow during clot retrieval per the instructions of the manufacturer. ${ }^{8}$

The Penumbra 054 aspiration system, consisting of a reperfusion catheter, separator, aspiration tubing, and suction pump, was set up for clot removal. A 7F guide catheter was positioned in the proximal ICA, and the reperfusion catheter was advanced to the proximal aspect of the occlusion. The vacuum pump was operated, and the separator was inserted into the reperfusion catheter. The side port of the rotating hemostasis valve was connected to the aspiration tubing. During aspiration, the separator was advanced and retracted to assist with clot removal.

The waveguide system was composed of a function generator, a transducer, and a thrombectomy wire $(156 \mathrm{~cm}$ in length and $0.4 \mathrm{~mm}$ in diameter). A $6 \mathrm{~F}$ guide catheter was positioned in the ICA, and through this, a $2.8 \mathrm{~F}$ microcatheter was positioned across the occlusive clot. The thrombectomy wire was delivered through the microcatheter, which was then withdrawn to expose the active section of the wire. The piezoelectric transducer was coupled on the proximal end to the wire, which causes high-frequency vibration to the distal active section. The mechanical motion of the active section produced cavitation streaming, which was necessary to fracture the fibrin matrix of the clot. The system was operated within the clot for 20 minutes.

Six experiments were performed for each device, and the experimental sequence was randomized. The maximum number of thrombectomy attempts was 3 . The balloon-guide catheter was positioned 


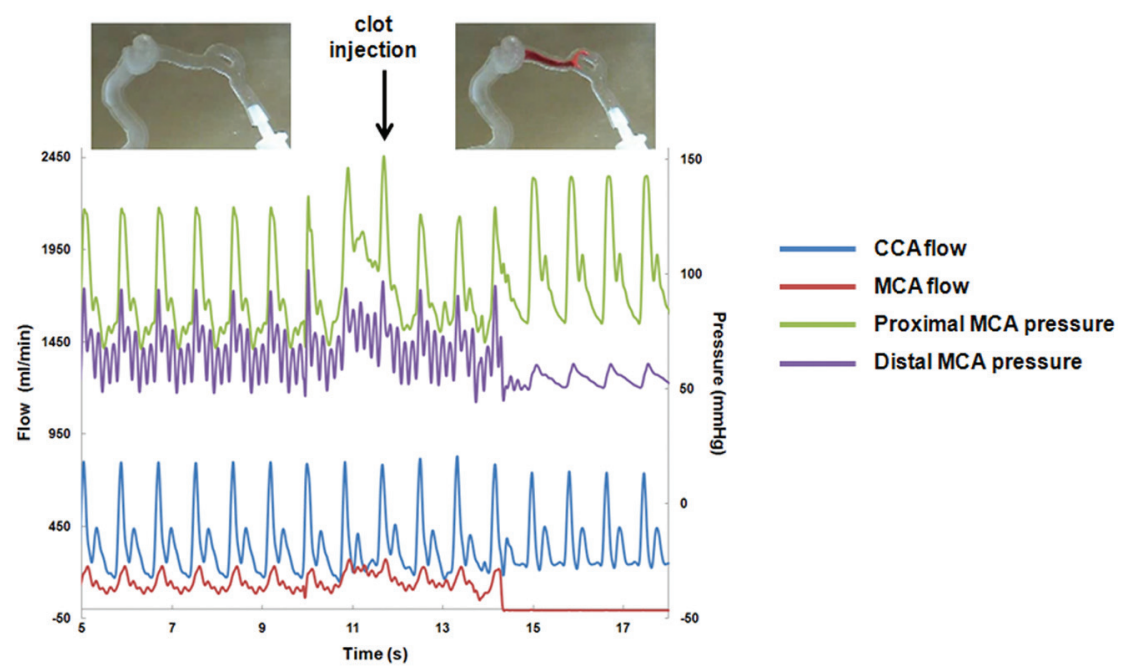

Fig 2. Variations of the hemodynamic variables before and after clot introduction.

in the ICA to temporarily arrest the flow during the clot removal when using the Merci retriever $(n=6)$ and the Solitaire FR $(n=5)$. One Solitaire test was excluded from data analysis due to the failure of proximal flow arrest. All procedures were performed under x-ray guidance (Allura FD20; Philips Healthcare, Best, the Netherlands) by the senior interventional neuroradiologist ( $>20$ years of experience).

\section{Efficacy and Safety End Points}

The primary efficacy end point was the amount of blood flow restored, which was defined as a ratio of flow in the ICA/MCA replica postprocedurally divided by the flow before creation of the occlusion. Follow-up angiography was performed to confirm the vessel patency for determination of the recanalization rate as the secondary efficacy end point.

The primary safety end point was the number and size of the clot fragments within the effluent of the ICA loop during MET. To measure the particulate size and size distribution, a Coulter counter (Beckman Multisizer 4, Brea, California) was used. In each experiment before the occlusion, $400 \mathrm{~mL}$ of blood-mimicking solution was collected as a blank for particle analysis. The blank sample accounted for microscopic background particles that were present in the flow loop. During MET, clot particulates with size between 200 and 1200 $\mu \mathrm{m}$ and 10 and $240 \mu \mathrm{m}$ were measured by the 2000 and $400 \mu \mathrm{m}$ apertures, respectively. Therefore, using 2 different apertures allowed complete particulate characterization of fragments within the range of 10-1200 $\mu \mathrm{m}$. A large volume of blood-mimicking solution was required for each analysis with the use of a $2000-\mu \mathrm{m}$ aperture. Therefore, the number of particle analysis tests was determined depending on the volume of solution collected during each experiment (between 1 and 2 analyses performed per experiment). Up to 5 replicate particulate tests were conducted by using the smaller $400-\mu \mathrm{m}$ aperture.

\section{Statistical Analysis}

All data were shown as mean \pm standard error of the mean. To compare the results from the particulate measurements, we used a nonparametric 1-way analysis of variance (Kruskal-Wallis) with Dunn posttests (Prism 5; GraphPad, LA Jolla, California). This method of analysis makes no assumption about the distribution of the data. Statistical significance was set at $P<.05$.

\section{Results}

\section{Flow Restoration}

Before occlusion, CCA and MCA flows were $363 \pm 1.9 \mathrm{~mL} /$ min and $132 \pm 1.4 \mathrm{~mL} / \mathrm{min}$, respectively (physiologic CCA flow, $378-450 \mathrm{~mL} / \mathrm{min}$; MCA flow, $117-152 \mathrm{~mL} / \mathrm{min}^{14}$ ). The pressure gradient between the proximal and distal MCA was $21 \pm 0.7 \mathrm{~mm} \mathrm{Hg}$. After occlusion, an increase in pressure gradient was observed ( $44 \pm 2.4 \mathrm{~mm} \mathrm{Hg}$ ), and complete MCA occlusion was confirmed (Fig 2).

The Merci retriever, Solitaire FR, and Enterprise stent were all able to engage the clot after deployment. To successfully restore flow, the clot slowly traveled through the ICA and was aspirated into the guide catheter (Fig $3 A-D$ ). In some experiments, the clot detached from the device when passing the tortuous ICA siphon, resulting in partial or failed thrombectomy (Fig $4 A-D$ ). Overall, the Solitaire FR achieved the highest recanalization rate $(100 \%)$ followed by the Merci retriever $(67 \%)$ and Enterprise stent $(17 \%)$. The average number of passes with the Solitaire FR, Merci retriever, and Enterprise stent was 1.2, 1.8, and 2.3, respectively. On average, the Solitaire FR restored $92.4 \pm 4.5 \%$ of flow. The Merci retriever restored $100 \%$ of flow in 4 experiments; however, in 2 experiments, the retriever was not able to ensnare the clot fragments during clot retrieval, resulting in a large portion of clot retained at the MCA bifurcation. Failure to recanalize the model was recorded in these 2 experiments. Flow restoration (29.2 \pm $6.4 \%$ ) was measured when the Enterprise stent was deployed within the clot, creating a temporary endovascular bypass. In 1 of the 6 experiments performed with the Enterprise stent, $86.6 \%$ flow was restored when part of the clot was removed by the device.

When the Penumbra aspiration system was used (Fig 5A$D)$, the continuous aspiration-debulking process produced visible clot particulates (circle in Fig 5B). After 2-3 passages of the aspiration device through the occluded area, $100 \%$ flow restoration was seen in 2 tests, partial flow restoration was measured in 3 tests, and no flow restoration was recorded in 1 test. Overall, the use of the Penumbra aspiration system showed a recanalization rate of $83 \%$ and an average of $92.5 \pm$ $4.6 \%$ flow restoration. Activation of the ultrasound waveguide 

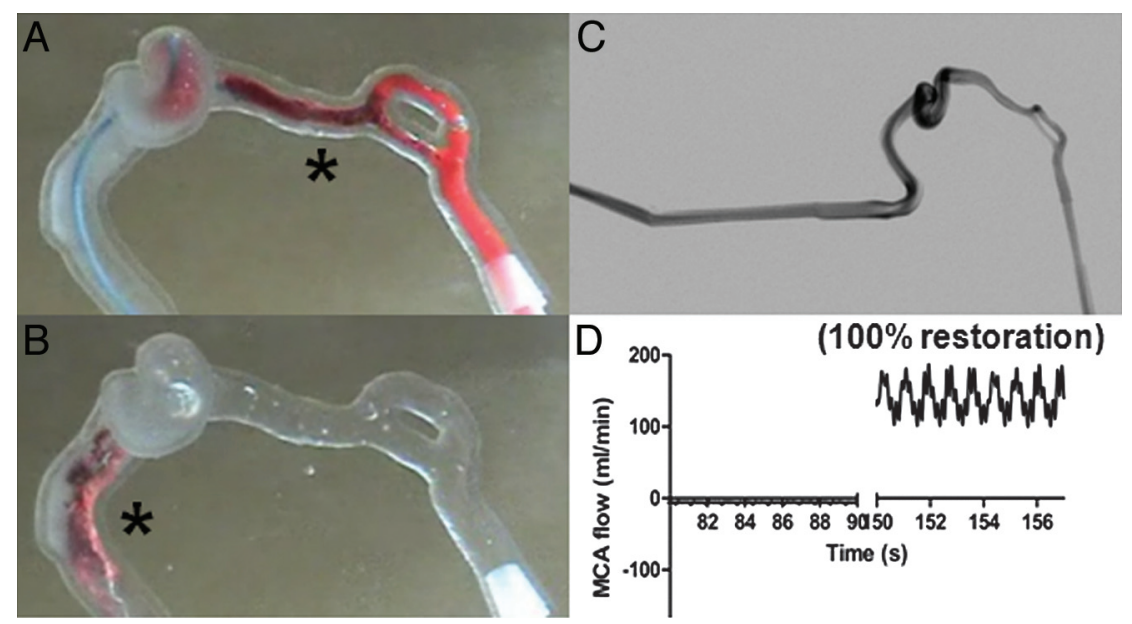

Fig 3. A, The Solitaire FR (asterisk) is deployed within the clot. Successful clot mobilization by the Solitaire FR is demonstrated in B. C, Flow restoration is presented on the postprocedure angiogram. $D$, The flow sensor detects complete recanalization.
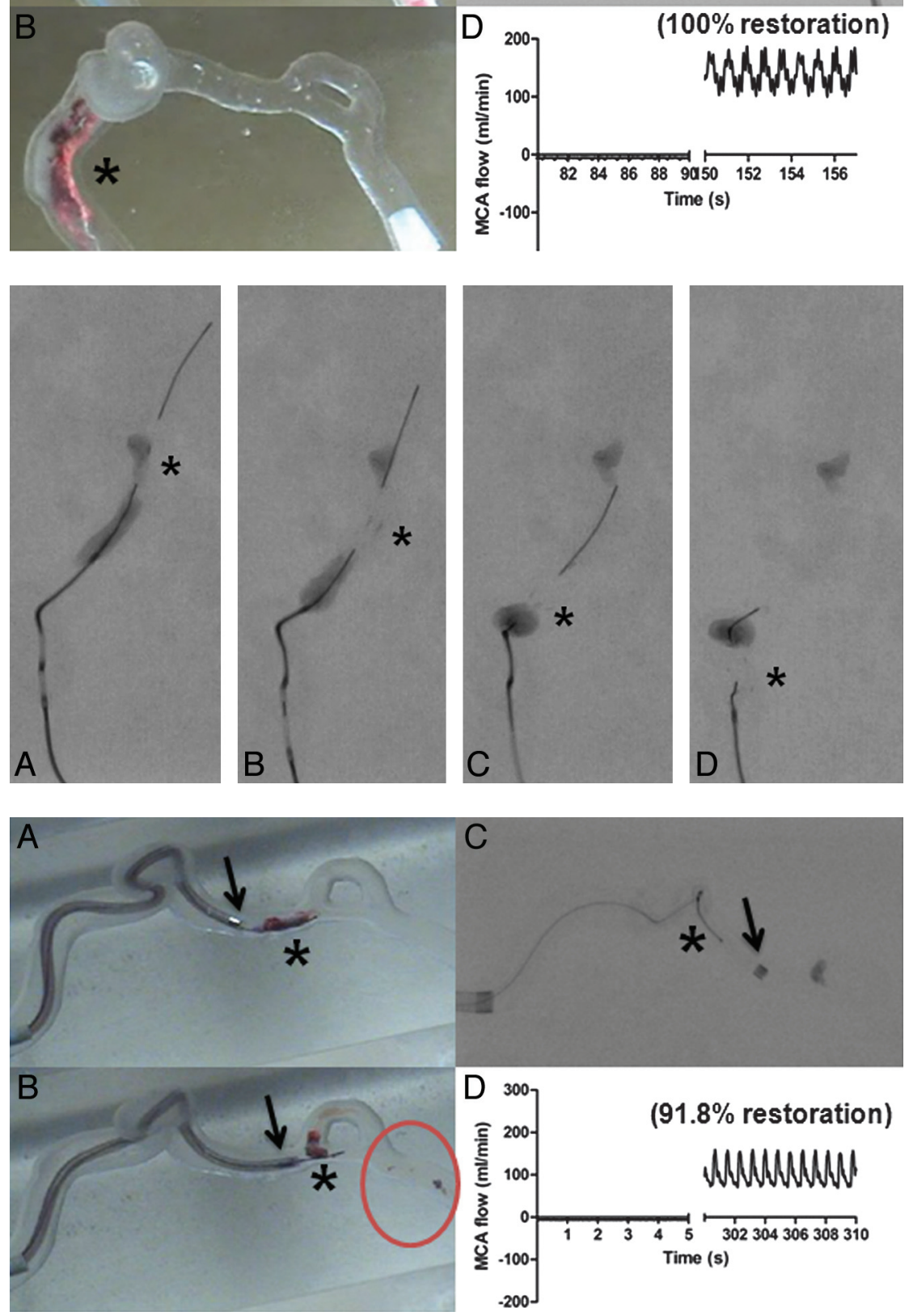

Fig 4. $A$, The clot is encased by the Enterprise stent struts after partial deployment. $B$, Clot is disrupted in 2 pieces, and 1 is retrieved along with the stent. $C$ and $D$, The retrieved clot is not retained within the stent struts when traveling through the ICA siphon. The asterisk indicates the distal radiopaque markers of the Enterprise stent.

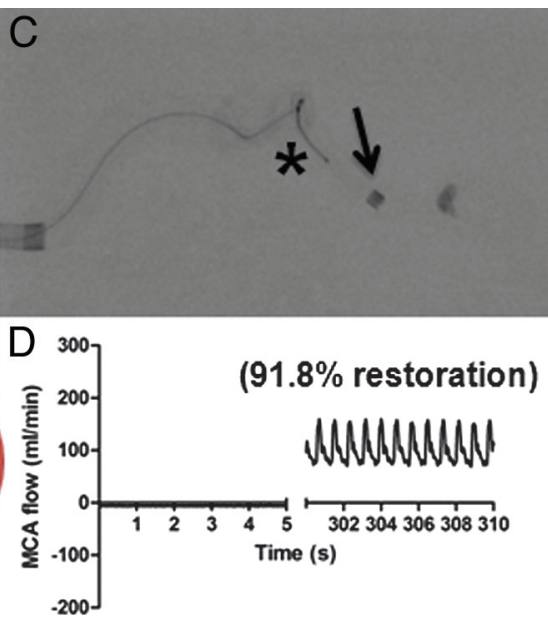

Fig 5. Partial recanalization of the MCA by using the Penumbra aspiration system. $A$, The reperfusion catheter (arrow) is placed at the proximal end of the clot. $B$, The clot is aspirated into the aspiration catheter with the assistance of the separator (asterisk). Small clot fragments (circle) are generated during the procedure. $C, A$ small portion of clot is retained at 1 of the MCA branches. D, Partial flow restoration is achieved.

failed to clear the occlusion. Although a blood plume was observed and small clot particles were generated during the activation of the waveguide, no flow restoration was achieved. The recanalization rate and flow restoration of each device (except the waveguide) are presented in Fig $6 A,-B$, respectively.

\section{Particulate Analysis}

The blank-subtracted results showed that thousands of small clot fragments with sizes smaller than $240 \mu \mathrm{m}$ were released by each device, and most particulates $(80.6 \%-92.1 \%$, depending on the device used) measured between 10 and $50 \mu \mathrm{m}$. The $2000-\mu \mathrm{m}$ aperture detected that $>90 \%$ of large clot debris had a size between 200 and $400 \mu \mathrm{m}$. The mean size of the small and large clot fragments was between $23 \pm 2.9 \mu \mathrm{m}$ and $37 \pm 5.1$ $\mu \mathrm{m}$ and $215 \pm 5.4$ and $285 \pm 26.0 \mu \mathrm{m}$, respectively, depending on the device used (Fig $6 C,-D$ ). There was a significant increase in the number of large clot fragments with the Penumbra aspiration system compared with the Merci retriever $(P<.05)$. The Penumbra aspiration device generated more fragments and the Solitaire FR produced larger fragments than the waveguide 

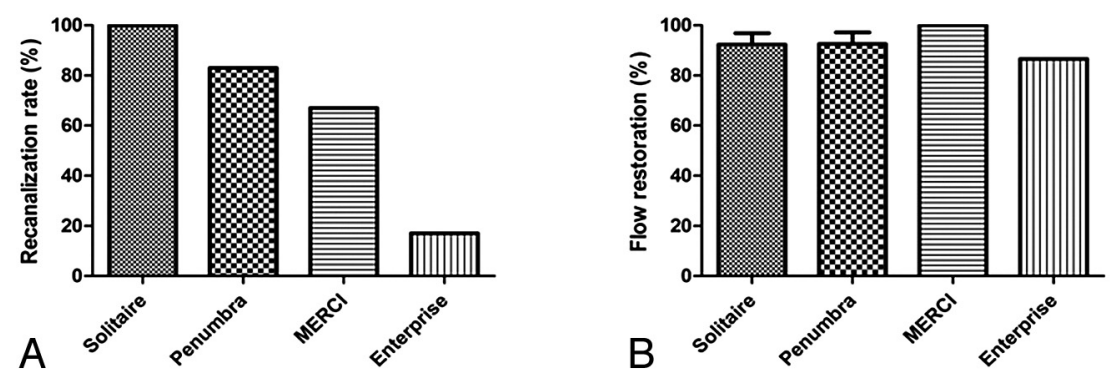

Fig 6. Recanalization rate $(A)$ and amount of flow restored $(B)$ with the use of Solitaire FR, Penumbra aspiration system, Merci retriever, and Enterprise stent are shown. Quantitative results of fragment number and size are presented in $C$ and $D$, respectively. Error bars are the standard error of the mean, and the asterisk indicates $P<.05$.

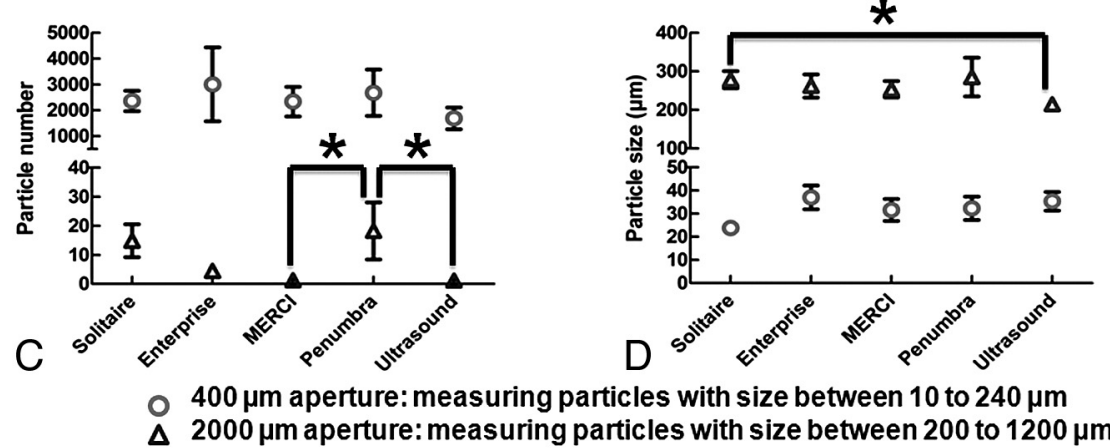

$(P<.05)$. However, because the waveguide failed to restore flow, this result was expected.

\section{Discussion}

In this study, the presented in vitro model system of cerebrovascular occlusion offers a reproducible testing environment to evaluate existing devices and/or newly developed prototypes. Performing MET in the aforesaid model system allows one to quantitatively determine the flow restoration and generation of clot fragments in the effluent. Currently, the existing in vitro experimental occlusion models for MET testing typically use stenosed straight tubing with clots prepared by using a variety of methods. ${ }^{15-18}$ The straight tubing does not reproduce the anatomic setting of the human cerebrovasculature. To improve these shortcomings, we selected the ICA siphon with moderate tortuosity from a patient population. It was built to allow realistic device tracking and was the key feature of the proposed ICA/MCA replica.

The similarities between mechanical properties of the human sources of cerebral emboli ${ }^{19}$ and the laboratory clot models $^{16-18,20}$ are often ignored. The radiopaque bovine clot model used in this work mimicked aged thromboemboli from atherosclerotic plaques and was selected for creation of MCA occlusions due to its low elasticity and hence high propensity for fragmentation. This enabled the evaluation of MET in a worstcase clinical scenario. Due to the variability in mechanical properties of thromboemboli from different sources, ${ }^{12}$ testing with 1 clot model is a limitation of this study. Future work should continue to evaluate the efficacy and safety envelope of each device technology on the basis of embolus characteristics. Thereafter, it may be possible to select the most appropriate thrombectomy device on the basis of clues of embolus composition, perhaps deduced from clinical evidence of the source of embolism or with imaging. ${ }^{21}$

The effectiveness of the Penumbra aspiration system, Merci retriever, and Solitaire FR in the described experimental occlusion model was consistent with that demonstrated in the previous clinical trials. The Penumbra Pivotal Stroke Trial showed a recanalization (Thrombolysis in Myocardial Infarction grade 2 or 3 ) rate of $81.6 \%,{ }^{5}$ which was nearly the same as that in our experiment (83\%). Similarly, the observed recanalization rate in this model system when using the Merci retriever $(67 \%)$ or Solitaire FR (100\%) was comparable with that reported in the Multi-MERCI registry $(57.3 \%)^{4}$ and a recent case series that used the Solitaire $(83-90 \%) .{ }^{26}$ These results may serve as evidence in support of the role of the proposed occlusion model in realistic assessment of device efficacy.

Mechanical clot retrieval was conducted by using the Merci retriever, Solitaire FR, and Enterprise stent in this study. Among these 3 groups, it was more frequent to find the disengagement of clot from the Enterprise stent during the retrieval process, in particular when clot traveled through the ICA siphon where higher tortuosity and larger vessel diameter were encountered. Notably, this device was not designed as a clot retriever. The resulting low recanalization rate of the Enterprise stent $(17 \%)$ could be attributed to the lack of proximal flow control without the use of a temporary occlusion of the ICA. As a consequence of this limitation, the continuous antegrade flow in the system tended to carry the clot downstream.

The presence of realistic siphon tortuosity may be, in part, responsible for the unsuccessful recanalization of the waveguide driven thrombectomy. Clot disruption caused by the waveguide in straight tubing was demonstrated in previous studies. ${ }^{9,18,22}$ Our experience (data not shown) is that the device led to clot ablation due to the high-frequency vibration of the distal active section when tested in straight or mildly tortuous tubing. However, when the same technique was applied to the ICA/MCA occlusion, no flow restoration was seen. Therefore, it was reasonable to assume that the tortuosity of the cerebrovasculature impeded the ultrasound wave propagation and, in part, accounted for the failure to restore flow. In clinical practice, intra-arterial mechanical techniques are very often used in conjunction with a thrombolytic agent such as 
urokinase or tPA; these combinations may have an enhanced effect on revascularization. However, in past research with the waveguide device in vitro, the addition of tPA did not improve recanalization effectiveness. ${ }^{18}$

Our in vitro model uses a blood analog solution consisting of water and glycerin, which matches the rheologic properties of blood under the flow conditions of our experiment. Although this creates an accurate hemodynamic environment, this model is not easily amendable to the use of thrombolytics due to the lack of circulating plasminogen. Although adjunctive pharmacologic thrombolysis may have produced some benefit in this model, the results would be difficult to translate into clinical practice due to the limitation imposed by the use of a blood analog solution.

Size and size distribution of the clot particulates released during the thrombectomy procedure described the potential risk of distal embolic shower. The Coulter principle was applied in 2 prior ex vivo studies to count and size the embolic fragments from the human atherosclerotic plaques that were released with ex vivo carotid angioplasty, ${ }^{23,24}$ and the authors concluded that hundreds of thousands of microemboli were generated during angioplasty. In this study, we calculated and compared the mean number of clot fragments in the effluent following MET with different devices. Similarly, thousands of microemboli were found in every $400 \mathrm{~mL}$ of blood-mimicking solution analyzed. It is critical to note that part of these fragments may be introduced into the system during the course of the experiment. Although every effort was made to reduce introduction of the particulates and subtraction of the blank samples acquired immediately before each experiment was performed, this study was not conducted in a clean room, and as such, this is a limitation of the research presented. However, the large particles generated were confirmed by visual inspection to be clot fragments. In our model system, the Penumbra aspiration catheter generated more large particles than the Merci system. This finding potentially explains the contradictory clinical evidence that despite the high recanalization rate realized with this device, the favorable clinical outcomes are reportedly less frequent ${ }^{5}$ than with either the Solitaire ${ }^{8}$ or Merci ${ }^{4}$ devices.

Although the risk of embolic shower calculated by using this in vitro model system is an important safety metric, vascular response and potential injury to the device used are of paramount importance. These cannot be assessed by using in vitro modeling, and consequently preclinical assessment in animal models is still required. ${ }^{25}$

\section{Conclusions}

In this study, the presented in vitro model system of cerebrovascular occlusion offers a reproducible testing environment to quantitatively evaluate existing devices and/or newly developed prototypes, which cannot be precisely measured in current animal models. The results showed that the recanalization rate of the thrombectomy device was related to the ability of the device to capture the clot during removal and the geometry of the cerebrovasculature. The risk of embolic shower was influenced by the mechanism of action for the thrombectomy device. As more devices become available, testing in such a preclinical model system may provide an efficient means for device development.
Disclosures: Ajay Wakhloo-RELATED: Grant. NIH, ${ }^{*}$ UNRELATED: Consultancy. Codman Neurovascular, Surpass Med, Stryker Neurovascular, Comments: fee per hour consultation; Grants/Grants Pending. Philips Healthcare, ${ }^{*} \mathrm{NIH},{ }^{*}$ Expert Testimony. Cohn Lifand Pearlman Herrmann \& Knopf, Stock/Stock Options: Surpass Med. Matthew Gounis-RELATED: Grant. NIH, ${ }^{*}$ UNRELATED: Consultancy. Micrus Endovascular, Soteira, Codman Neurovascular, Comments: Fee per hour consultation, Grants/Grants Pending. Boston Scientific, ${ }^{*}$ Concentric Medical, ${ }^{*}$ Codman Neurovascular, ${ }^{*}$ Covidien, ${ }^{*}$ ev3 Neurovascular, ${ }^{*}$ Geurbet, ${ }^{*}$ Neuravi, ${ }^{*}$ Neurointerventional Technologies, ${ }^{*}$ Thrombolytic Science, ${ }^{*}$ Stryker Neurovascular, ${ }^{*}$ Sanofi-Aventis. ${ }^{*}$ Money paid to the institution.

\section{References}

1. Baron JC, von Kummer R, del Zoppo GJ. Treatment of acute ischemic stroke: challenging the concept of a rigid and universal time window. Stroke 1995;26:2219-21

2. Gralla J, Brekenfeld C, Arnold M, et al. Acute stroke: present and future of catheter-based interventions. Herz 2008;33:507-17

3. Saver JL. Time is brain-quantified. Stroke 2006;37:263-66

4. Smith WS, Sung G, Saver J, et al. Mechanical thrombectomy for acute ischemic stroke: final results of the Multi MERCI trial. Stroke 2008;39:1205-12

5. Penumbra Pivotal Stroke Trial Investigators. The Penumbra Pivotal Stroke Trial: safety and effectiveness of a new generation of mechanical devices for clot removal in intracranial large vessel occlusive disease. Stroke 2009:40:2761-68

6. Wakhloo AK, Gounis MJ. Retrievable closed cell intracranial stent for foreign body and clot removal. Neurosurgery 2008;62:390-93

7. Ansari S, McConnell DJ, Azari H, et al. Impact of intracranial self-expanding stents in the treatment of acute ischemic stroke: efficacy and limitations. J Neurointerv Surg 2011 Feb 10. [Epub ahead of print]

8. Castano C, Dorado L, Guerrero C, et al. Mechanical thrombectomy with the Solitaire AB device in large artery occlusions of the anterior circulation: a pilot study. Stroke 41:1836-40

9. Ariani M, Fishbein MC, Chae JS, et al. Dissolution of peripheral arterial thrombi by ultrasound. Circulation 1991;84:1680-88

10. Goldberg SN, Ahmed M, Weinstein J, et al. Low-power transverse ultrasonic treatment of portal vein thrombosis in an animal model. J Vasc Interv Radiol 2002;13:915-21

11. Chueh JY, Wakhloo AK, Gounis MJ. Neurovascular modeling: small-batch manufacturing of silicone vascular replicas. AJNR Am J Neuroradiol 2009;30:1159-64

12. Chueh JY, Wakhloo AK, Hendricks GH, et al. Mechanical characterization of thromboemboli in acute ischemic stroke and laboratory embolus analogs. AJNR Am J Neuroradiol 2011;32:1237-44

13. Hirata K, Yaginuma T, O'Rourke MF, et al. Age-related changes in carotid artery flow and pressure pulses: possible implications for cerebral microvascular disease. Stroke 2006;37:2552-56

14. Blankensteijn JD, van der Grond J, Mali WP, et al. Flow volume changes in the major cerebral arteries before and after carotid endarterectomy: an MR angiography study. Eur J Vasc Endovasc Surg 1997;14:446-50

15. Grimm J, Jahnke T, Muhle C, et al. Influence of thrombus age on the mechanical thrombectomy efficacy of the amplatz thrombectomy device in vitro. Cardiovasc Intervent Radiol 2003;26:265-68

16. Krueger K, Deissler P, Coburger S, et al. How thrombus model impacts the in vitro study of interventional thrombectomy procedures. Invest Radiol 2004;39:641-48

17. Muller-Hulsbeck S, Grimm J, Leidt J, et al. Comparison of in vitro effectiveness of mechanical thrombectomy devices. J Vasc Interv Radiol 2001;12:1185-91

18. Salazar GM, Faintuch S, Gladstone SR, et al. In vitro analysis of downstream particulates with mechanical thrombectomy devices: comparison of $20-\mathrm{kHz}$ sonothrombolytic and rotating dispersion wire systems. J Vasc Interv Radiol 2009;20:634-39

19. Marder VJ, Chute DJ, Starkman S, et al. Analysis of thrombi retrieved from cerebral arteries of patients with acute ischemic stroke. Stroke 2006;37: 2086-93

20. Asakura F, Yilmaz H, Abdo G, et al. Preclinical testing of a new clot-retrieving wire device using polyvinyl alcohol hydrogel vascular models. Neuroradiology 2007;49:243-51

21. Liebeskind DS, Sanossian N, Yong WH, et al. CT and MRI early vessel signs reflect clot composition in acute stroke. Stroke 2011;42:1237-43

22. Hong AS, Chae JS, Dubin SB, et al. Ultrasonic clot disruption: an in vitro study. Am Heart J 1990;120:418-22

23. Coggia M, Goeau-Brissonniere O, Duval JL, et al. Embolic risk of the different stages of carotid bifurcation balloon angioplasty: an experimental study. $J$ Vasc Surg 2000;31:550-57

24. Rapp JH, Pan XM, Yu B, et al. Cerebral ischemia and infarction from atheroemboli < 100 microm in size. Stroke 2003;34:1976-80

25. Mehra M, Henninger N, Hirsch JA, et al. Preclinical acute ischemic stroke modeling. J Neurointerv Surg 2012;4:307-13

26. Saver JL, Jahan R, Levy EI, et al. Preliminary results of the SOLITAIRE with the intention for thrombectomy (SWIFT) multicenter, randomized clinical trial. International Stroke Conference, February 3, 2012, New Orleans, Louisiana 